\title{
Bacterial Profile, Antimicrobial Susceptibility Pattern, and Associated Factors of Community- and Hospital-Acquired Urinary Tract Infection at Dessie Referral Hospital, Dessie, Northeast Ethiopia
}

\author{
Berhanu Adugna $\mathbb{D}^{1},{ }^{1}$ Bekele Sharew $\mathbb{D}^{2},{ }^{2}$ and Mohabaw Jemal $\mathbb{I D}^{3}$ \\ ${ }^{1}$ Dessie Referral Hospital, Laboratory Technologist, The Department of Medical Microbiology, Dessie, Ethiopia \\ ${ }^{2}$ Wollo University, Department of Medical Laboratory Sciences, Dessie, Ethiopia \\ ${ }^{3}$ University of Gondar, College of Medicine and Health Sciences, School of Biomedical and Laboratory Sciences, \\ Department of Medical Microbiology, Gondar, Ethiopia
}

Correspondence should be addressed to Mohabaw Jemal; mohabawjemal@gmail.com

Received 15 February 2021; Revised 27 August 2021; Accepted 4 September 2021; Published 20 September 2021

Academic Editor: Joseph Falkinham

Copyright (c) 2021 Berhanu Adugna et al. This is an open access article distributed under the Creative Commons Attribution License, which permits unrestricted use, distribution, and reproduction in any medium, provided the original work is properly cited.

\begin{abstract}
Background. Bacterial urinary tract infection is among the most common community and hospital-acquired infections. Therefore, to know the status of the community and hospital-acquired urinary tract infection, antimicrobial susceptibility patterns, and associated factors among urinary tract infection profiles are essential to physicians and health workers to implement appropriate intervention. Methods. An institution-based cross-sectional study was conducted among 422 urinary tract infection suspected patients. All isolates were identified by standard microbiological techniques, and their antibiotic susceptibility was done by the Kirby-Bauer disc diffusion method. Data were entered using EpiData version 3.1 and analyzed by SPSS software version $20 . P$ value $<0.05$ at $95 \%$ CI was considered statistically significant. Result. Of 422 urine samples processed, 100 (23.7\%) yielded bacterial isolates. About 50(30.7\%) and 50(19.3\%) were bacterial isolates from the community and hospitalized patients, respectively. E. coli 44/103(42.7\%) predominated across the two groups, followed by S. aureus 25/103(24.3\%), CONs, 14/103(13.5\%), Klebsiella spp. 7/103(6.78), Proteus spp. 3/103(2.91), and Enterococcus spp. 3/103 (2.91\%). Pseudomonas spp. 3/103 (2.91), Citrobacter spp. 2/103(1.94\%), and Acinetobacter spp. 1/103(0.999), which were isolated from only the hospitalized patients. Meropenem susceptibly was $100 \%$ in both study groups and Ampicillin resistance was documented as $83.3 \%$ to $100 \%$ and $76.9 \%$ to $100 \%$ in hospitalized and community-acquired samples, respectively. Conclusion. This study found a high prevalence of bacterial urinary tract infection in the study area and a high rate of bacterial resistance was observed to most antimicrobial drugs tested. Meropenem and nitrofurantoin were the most active drugs for urinary tract infections. Therefore, expanding routine bacterial culture and identification with antimicrobial susceptibility testing and strengthening regular surveillance systems are essential for appropriate patient care.
\end{abstract}

\section{Introduction}

Urinary tract infections (UTI) constitute a significant public health problem and present an important cause of morbidity and mortality worldwide $[1,2]$. Bacterial pathogens are the commonest etiological agent to cause UTI. It affects both lower and upper urinary tracts with different clinical symptoms, including fever, dysuria, urgency, burning sensation, and intermittent urination.

Suprapubic tenderness [3] is the second most common infection after respiratory tract infections and accounts for a great proportion of prescriptions of antibiotics. The major causative organisms for UTI are bacteria organisms. They account for more than $95 \%$ of cases $[4,5]$, which may be 
Gram-negative and Gram-positive bacteria that account for $80-85 \%$ and $15-20 \%$, respectively $[6,7]$. Urinary tract infection starts with contamination of the periurethral by uropathies residing in the bowel flora colonization. The urethra ascends to the bladder and migrates to the kidney or prostate. The result of host-pathogen complex interactions ultimately determines whether uropathogens are successful in colonization or eliminated [8]. Community-acquired UTI (CAUTI) is the member of intestinal microbial flora. The most common are E. coli and Klebsiella spp. In communityacquired urinary tract infection, E. coli and S. saprophyticus accounts for $80 \%$ and $5 \%$ to $15 \%$ of outpatients, respectively, across the various regions of the world, and the remaining $5 \%$ to $10 \%$ of cases are aerobic Gram-negative rods such as Klebsiella spp. and Proteus spp. and other Enterobacter spp. [9-11].

Hospital-acquired urinary tract (HAUTI) infections are mostly healthcare-related infections because these are the ones that occur more frequently and are commonly related to the use of a medical device such as catheterization $[10,12,13]$.

Urinary tract infections are an important cause of septicemia, resulting in high mortality rates, prolonged hospital stays, and increased healthcare costs [14]. The death of hospitalized patients among the victims of hospital-acquired urinary tract infections are 2 to 3-times higher than those among nonbacteriuric patients [15]. Catheter-associated urinary tract infections occur with high incidence if healthcare safety is not maintained. Studies reveal that $79.3 \%$ of UTI can be prevented if catheterization is not performed in hospitals [16]. Multiple risk factors can affect the occurrence of urinary tract infections. These include age, sex, catheterization and hospitalization, previous exposure to antibiotics, recurrent UTI, duration of catheterization, and care of catheter [12, 17-20].

In developing countries, including Ethiopia, where there is a high level of poverty and poor hygiene practices, there is also a high prevalence of fake and spurious drugs of questionable quality in circulation $[21,22]$. Besides this, the easy availability in the community without clinician order and low cost makes the drugs subject to abuse. These make increasing drug resistance [23]. Therefore, the current study aims to assess the prevalence and antimicrobial susceptibility pattern and associated factors among urinary tract infection profiles and provides updated information to regulatory bodies and those who would like to use the findings for the development of intervention strategies as appropriate.

This manuscript is presented in the < research square $>$ as a preprint. https://www.researchsquare.com/ article/rs-157817/v1.

\section{Materials and Methods}

2.1. Study Design and Setting. A hospital-based cross-sectional study was conducted at Dessie Referral Hospital from March 2019- April 2019. The hospital was found in Dessie town with a distance of $401 \mathrm{~km}$ from the capital city of Ethiopia, Addis Ababa, and $471 \mathrm{~km}$ far from Bahir Dar, which is the capital city of Amhara Regional State. The hospital provides health services for more than 6 million people. This large number of people from the surrounding zones and nearby regions visits the hospital for different medical services. Dessie Referral Hospital provides emergency, ART services, chronic care, surgical, dental, medical, pediatric, gynecologic, obstetric, and other services.

2.2. Sample Size and Sampling Technique. A single population proportion formula was used to determine the sample size, $50 \%$ prevalence (anticipated proportion). By considering a 5\% margin of error, $95 \%$ confidence level, and $10 \%$ nonresponse rate, a total of 422 participants were proposed and systematically recruited.

\subsection{Data Collection Tools and Procedure}

2.3.1. Sociodemographic and Clinical Data. Data related to sociodemographic factors, clinical data, and associated factors were collected using pretested structured and standardized questionnaires from reviewed literature. They were prepared in English, translated to the local language (Amharic), and then translated back into English to check its consistency. Data were collected from the places of study participants from outpatient departments or inpatient wards.

2.4. Sample Collection and Transportation. A freshly voided midstream urine sample $(10-15 \mathrm{ml})$ and catheterized urine samples were collected using a sterile container with screw cap tops. Urine samples were examined chemically and microscopically. Then, they were delivered to Amhara public health microbiology laboratory and processed within 12 hours for analysis. In case of delay, the samples were re-

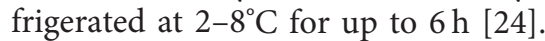

2.5. Isolation and Identification Procedure. Urine specimens collected from different departments were directly inoculated by using calibrated inoculating wire loop $(0.001 \mathrm{~mL})$ on cystine lactose electrolyte deficient agar (CLED) (Oxoid Ltd., England). Culture plates were incubated in the aerobic environment at $370 \mathrm{C}$ for $24 \mathrm{hrs}$. After incubation, all suspected colonies were subcultured onto MacConkey agar (Oxoid, England) and 5\% sheep blood agar (Oxoid, England) for further identification. All positive cultures were further identified by their colony characteristics, and Gram staining was done to identify Gram-positives from Gramnegatives. The biochemical tests used for final identification were Triple sugar iron agar test, Sulphide Indole production test, citrate utilization test, urease production test, and catalase and coagulase test [24].

2.6. Antimicrobial Susceptibility Testing. Antimicrobial susceptibility tests were carried out using the Kirby-Bauer disc diffusion method as per the Clinical Laboratory Standards Institute (CLSI) guidelines on Muller-Hinton agar. Pure colonies were taken from plates with fresh, pure culture using sterile wire loops and transferred to a tube containing 
$5 \mathrm{ml}$ of $0.85 \%$ normal saline and mixed gently until it formed a homogeneous suspension. The turbidity of the suspension was then adjusted to the density of a McFarland standard 0.5 to standardize the size of the inoculum. The surface of the Muller-Hinton agar was then completely covered by rotating the swab with the suspension. The plates were allowed to dry for 3-5 minutes: then, discs were evenly distributed $24 \mathrm{~mm}$ apart on the inoculated plate using sterile forceps and incubated at $370 \mathrm{C}$ for 18-24 hours. The diameter of the zone of inhibition around the disc was measured using a ruler. Results were interpreted as Sensitive, Intermediate, and Resistant based on CLSI 2016 guideline [25]. The following routinely used antimicrobials were tested: ampicillin (AMP, $10 \mu \mathrm{g}$ ), amoxclavunic (20/10), tetracycline (TE, $30 \mu \mathrm{g}$ ), ciprofloxacin (CIP, $5 \mu \mathrm{g}$ ), trimethoprim + sulphamethozol (SXT, 1.25/23.75), gentamicin $(\mathrm{CN}, 10 \mu \mathrm{g})$, ceftriaxone (CRO, $30 \mu \mathrm{g})$, cefixime (CXM, $5 \mu \mathrm{g})$, nalidixic acid (NA, $30 \mu \mathrm{g}$ ), nitrofurantoin (F, $300 \mu \mathrm{g}$ ), piperacillin (PIP, $100 \mu \mathrm{g}$ ), vancomycin (VAN, $10 \mu \mathrm{g}$ ), penicillin (PEN, $10 \mu \mathrm{g}$ ), meropenem (MER, $10 \mu \mathrm{g}$ ), and tobramycin (TOB, $10 \mu \mathrm{g}$ ), and then plates were incubated at $37^{\circ} \mathrm{C}$ for $24 \mathrm{~h}$. Multidrug resistance was defined as the resistance of an isolate to three or more antimicrobial classes tested [25].

2.6.1. Data Quality Control. The data collector was trained in the methods of data collection technique. The completeness and clarity of the collected data were checked every day. A pretested structured questionnaire was used for the data collection on sociodemographic characteristics and associated risk factors. The questionnaire was initially prepared in English and translated into the local language, Amharic.

2.6.2. Laboratory Quality Control. The sterility of culture media was checked by incubating about $5 \%$ a batch of the media at 35-37OC overnight and evaluated for possible contamination. Standard reference strains of $S$. typhimurium (ATCC-14028) and E. coli (ATCC-25922) were used as quality control throughout the study for culture [26]. Data quality was ensured at various activities of the study by following the prepared standard operating procedure (SOP) of the laboratory.

2.7. Data Analysis and Interpretation. Collected data were entered into Epi-data 3.1 and exported to SPSS version 20 statistical software for analysis. During analysis, Descriptive statistics including mean, frequency, and percentage were used to summarize the data as appropriate. Then the findings of this study were presented in the form of texts, tables, and graphs as appropriate. A $P$ value of $<0.05$ was considered statistically significant.

2.7.1. Operational Definition. Community-acquired UTI is an infection if an individual with UTI is suspected before hospital admission and specimens are collected from the outpatient or within less than 48 hours of hospital admission.
2.7.2. Hospital-Acquired UTI. Those individuals are not present or incubating at the time of the hospital admission and developing 48-72 hours after hospital admission. This manuscript presents in the $<$ research square $>$ as a preprint. https://www.researchsquare.com/article/rs-157817/v1.

\section{Results}

3.1. Sociodemographic and Clinical Characteristics. A total of 422 Urinary tract infection suspected patients were included in this study. Of these, 281 (66.6\%) were female and 141 (33.4\%) were male. The age range of study participants was 5-90 years, with a median age of 32 years. Among the total number of study subjects, $114(27 \%), 110(26 \%)$, and 66 (15.6\%) were (30-44), (15-29), and (0-14) years of age, receptively. One hundred thirty-seven had an educational level of reading and writing only. The majority of the study subjects were from urban areas and had lower $(<500 \mathrm{EBR})$ monthly income, 238 (56.4\%) and $214(50.7 \%)$, respectively (Table 1). Moreover, 154 (36.4\%), 72 (17\%), 49 (11.6), and 40 (9.5\%) had a history of previous exposure to antibiotics, diabetics (CDs), history of renal calculi, and history of urinary tract obstruction in the community and hospitalacquired UTI, respectively. Out of the total participants clinically diagnosed with urinary tract infection were studied to isolate bacteria from urine, of which 259 (61.4\%) were from community-acquired cases and 163 (38.6\%) from hospital-acquired cases [Table 1].

3.2. Prevalence of Urinary Tract Infections. The overall prevalence of urinary tract infection was $23.7 \%(100 / 422)$ (95\% CI: 19.3-27.5). Out of 259 community-acquired UTI symptomatic patients, $19.3 \%$ (50/259) (95\% CI:16.0-24.7) were culture-positive and 30.7\% (50/163) (95\% CI:23.3-38) were culture-positive for hospitalized patients (Table 2). Of the total 422 urine specimens processed, $74.2 \%$ (313/422) showed no bacteriuria growth and 2.13\% (9/422) showed insignificant bacterial growth. Significant growth was present in $23.7 \%$ (100/422) samples with $22.99 \%$ (97/422) single growth and $0.71 \%(3 / 422)$ in mixed growth with two organisms in hospitalized patients (Figure 1). Three out of four hundred twenty-two (0.71\%) samples with two bacteria each were isolated, making the number of bacteria isolated to be 103 with the isolation rate of (24.4\%). From a total of 103 different uropathies bacterial isolated, 53 (51.46\%) were hospital-acquired setting isolates, and 50(48.54\% were community-acquired setting isolates (Table 3). Sixty-one (59.22\%) were Gram-negative bacilli and $42(40.78 \%)$ were Gram-positive cocci (Figure 2).

The predominant bacteria isolated in both community and hospital-acquired UTI were E. coli $52 \%(26 / 50)$ versus $33.96 \%$ (18/53), followed by S. aureus $24 \%(12 / 50)$ vs. $24.5 \%$ (13/53), CONs16\% (8/53) vs. $11.32 \%$ (6/53), Klebsiella spp. $4 \%(2 / 50)$ vs. $9.43 \%$ (5/53), Proetus spp. $2 \%$ (1/50) vs. $5.7 \%(3 /$ 53), and Enterococcus spp. $2 \%(1 / 50)$ vs. $3.8 \%(2 / 53)$ were isolated in both study groups whereas Pseudomonas spp. 5.7\% (3/53), Citrobacter spp. 3.8\% (2/53), and Acinetobacter spp. $1.79 \%(1 / 53)$ were isolated only in hospitalized patients (Table 3). 
TABLE 1: Sociodemographic characteristics of the study participant among the community and hospital-acquired UTI at Dessie Referral Hospital.

\begin{tabular}{|c|c|c|c|c|}
\hline \multicolumn{2}{|c|}{ Characteristics $(n=422)$} & \multirow{2}{*}{$\begin{array}{c}\text { Negative, } N(\%) \\
122(86.5)\end{array}$} & \multirow{2}{*}{$\begin{array}{c}\text { Positive, } N(\%) \\
19(13.5)\end{array}$} & \multirow{2}{*}{$\begin{array}{c}\text { Total, N (\%) } \\
141(33.4)\end{array}$} \\
\hline Sex & Male & & & \\
\hline sex & Female & $200(76.7)$ & $81(23.3)$ & $281(66.6)$ \\
\hline \multirow{5}{*}{ Age } & $0-14$ & $55(83.3)$ & $11(16.6)$ & $66(15.6)$ \\
\hline & $15-29$ & $77(70)$ & $33(30)$ & $110(26)$ \\
\hline & $30-44$ & $96(84.2)$ & $18(15.7)$ & $114(27)$ \\
\hline & $45-59$ & $60(83.3)$ & $12(16.7)$ & $72(17)$ \\
\hline & $>60$ & $34(56.6)$ & $26(43.3)$ & $60(14.2)$ \\
\hline \multirow{5}{*}{ Education } & Illiterate & $89(71.7)$ & $35(28.2)$ & $124(29.4)$ \\
\hline & Read and write only & $106(77.4)$ & $31(22.6)$ & $137(32.4)$ \\
\hline & Up to grades 8 complete & $86(81)$ & $20(19)$ & $106(25)$ \\
\hline & Up to grade 12 complete & $17(70)$ & $7(29)$ & $24(5.6)$ \\
\hline & University/college and above & $24(77.4)$ & $7(22.6)$ & $31(7.3)$ \\
\hline \multirow{2}{*}{ Residence } & Urban & $185(77.7)$ & $53(22.3)$ & $238(56.4)$ \\
\hline & Rural & $137(72.8)$ & $51(27.2)$ & $188(43.6)$ \\
\hline \multirow{3}{*}{ Monthly income } & Lower $(<500 \mathrm{EBR})$ & $150(70)$ & $64(30)$ & $214(50.7)$ \\
\hline & Medium (5001-1000) & $106(83.4)$ & $21(16.6)$ & $127(30)$ \\
\hline & Higher (>1001 EBE) & $66(81)$ & $15(8.5)$ & $81(19.3)$ \\
\hline \multirow{2}{*}{ Pregnancy status $(n=199)$} & Yes & $10(50)$ & $10(50)$ & $20(10)$ \\
\hline & No & $136(78.8)$ & $43(21.2)$ & $179(90)$ \\
\hline \multirow{2}{*}{ Diabetics (CDs) } & Yes & $29(40.2)$ & $43(59.7)$ & $72(17)$ \\
\hline & No & $293(83.7)$ & $57(16.3)$ & $350(83)$ \\
\hline \multirow{2}{*}{ History of urinary tract obstruction } & Yes & $17(42.5)$ & $23(57.5)$ & $40(9.5)$ \\
\hline & No & $305(80.9)$ & $77(19.1)$ & $382(90.5)$ \\
\hline \multirow{2}{*}{ Previous exposure to antibiotics } & Yes & $90(58.4)$ & $64(41.5)$ & $154(36.4)$ \\
\hline & No & $232(86.5)$ & $36(13.4)$ & $268(63.6)$ \\
\hline \multirow{2}{*}{ Recurrence urinary tract infection } & Yes & $107(64)$ & $60(36)$ & $167(40)$ \\
\hline & No & $215(89.1)$ & $40(10.9)$ & $255(60)$ \\
\hline \multirow{2}{*}{ History of renal calculi } & Yes & $38(77.5)$ & $11(22.4)$ & $49(11.6)$ \\
\hline & No & $303(81.4)$ & $70(18.6)$ & $373(88.4)$ \\
\hline \multirow{2}{*}{ Contraceptive method $(n=253)$} & Yes & $16(84.2)$ & $3(15.8)$ & $19(7.5)$ \\
\hline & No & $178(78.3)$ & $56(21.7)$ & $234(92.5)$ \\
\hline \multirow{2}{*}{ Waiting time in hospital $(n=163)$} & $48-72$ hours & $45(80.4)$ & $11(19.6)$ & $56(34.3)$ \\
\hline & $>72$ hours & $68(63.6)$ & $39(36.4)$ & $107(65.7)$ \\
\hline \multirow{2}{*}{ History of catheterization $(n=163)$} & Yes & $21(41.2)$ & $30(58.8)$ & $51(31.2)$ \\
\hline & No & $92(82.1)$ & $20(17.9)$ & $112(68.8)$ \\
\hline \multirow{2}{*}{ Duration of catheterization $(n=53)$} & $<$ One week & $8(53.3)$ & $7(46.7)$ & $15(28.3)$ \\
\hline & $>$ One week & $13(34.2)$ & $25(65.8)$ & $38(71.7)$ \\
\hline
\end{tabular}

TABle 2: Distribution of culture among community versus hospital-acquired UTI groups of patients at Dessie Referral Hospital, Dessie.

\begin{tabular}{lcccc}
\hline UTI types & Urine cultured & Cultured positive cases & Percentage & $X^{2}$ \\
\hline CAUTI & 259 & 50 & 19.3 & \\
HAUTI & 163 & 50 & 30.7 & 6.537 \\
Total & 422 & 100 & 23.7 & 0.011 \\
\hline
\end{tabular}

Note: by chi-squire, $X^{2}=6.537, \mathrm{df}=1, P=0.011$; HAUTI: hospital-acquired UTI; CAUTI: community-acquired UTI.

The frequency of isolated bacteria increases the duration of catheterization. E. coli, S. aureus, CONs, Pseudomonas spp., Klebsiella, and Enterococcus spp. were increased after one week of catheterization. However, Proteus spp., Citrobacter spp., and Acinetobacter spp. were found only for more than one week of catheterization (Figure 2).
3.3. Risk Factors of Community and Hospital-Acquired Urinary Tract Infection. In bivariate and multivariate analysis of CAUTI study subject, the previous usage of antibiotics was 4.427 times more likely to have developed urinary tract infection when compared with nonusers of antibiotics (AOR = 4.427; CI, 1.214-16.146, $P=0.024)$. However, there 


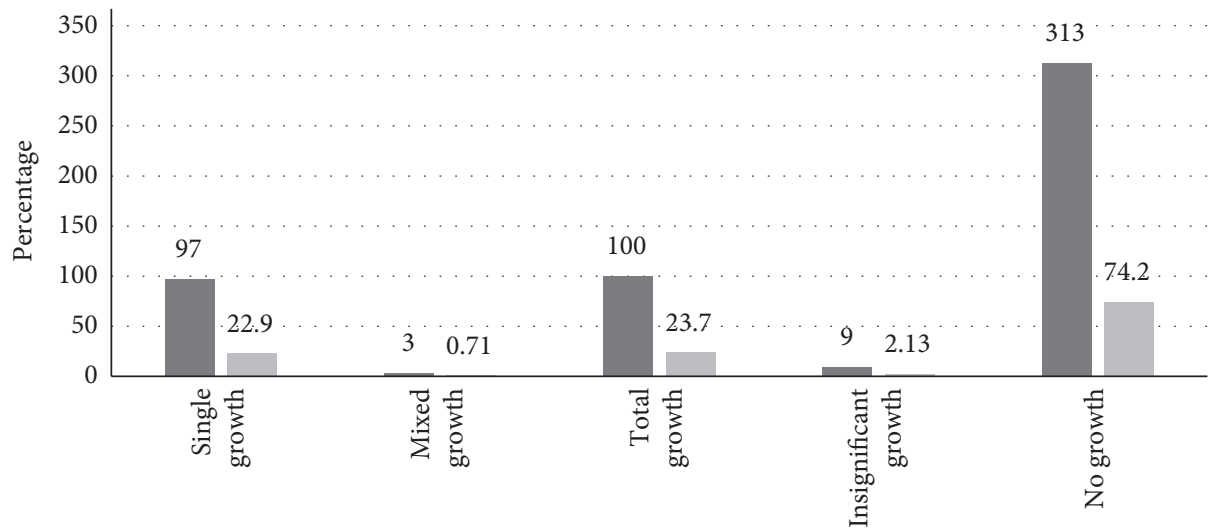

Results of growth in culture

- Number

1- Percentage

Figure 1: Bacterial growth results in the culture media from a total urine sample processed at Dessie Referral Hospital, Dessie.

Table 3: Distribution of bacterial isolated among CAUTI and HAUTI at Dessie Referral Hospital Dessie.

\begin{tabular}{|c|c|c|c|c|c|}
\hline \multirow{3}{*}{ Isolated organism } & \multicolumn{5}{|c|}{ Type of UTI } \\
\hline & \multicolumn{2}{|c|}{ CAUTI $(n=50)$} & \multicolumn{2}{|c|}{ HAUTI $(n=53)$} & \multirow{2}{*}{ Total } \\
\hline & Male & Female & Male & Female & \\
\hline Escherichia coli & $5(19.2)$ & $21(80.8)$ & $4(22.2)$ & $14((77.8)$ & $44(42.72)$ \\
\hline S. aureus & $3(25)$ & $9(75)$ & $3(23)$ & $10(77)$ & $25((24.27)$ \\
\hline CONS & $1(12.5)$ & $7(87.5)$ & $1(16.7)$ & $5(83.3)$ & $14(13.6)$ \\
\hline Klebsiella spp. & $1(50)$ & $1(50)$ & $1(20)$ & $4(80)$ & $7(6.78)$ \\
\hline Proteus spp. & $0(0)$ & $1(100)$ & $0(0)$ & $3(100)$ & $4(3.88)$ \\
\hline Enterococcus spp. & $0(0)$ & $1(100)$ & $0(0)$ & $2(100)$ & $3(2.91)$ \\
\hline Pseudomonas spp. & $0(0)$ & $0(0)$ & $0(0)$ & $3(100)$ & $3(2.91)$ \\
\hline Citrobacter spp. & $0(0)$ & $0(0)$ & $0(0)$ & $2(100)$ & $2(1.94)$ \\
\hline Acinetobacter spp. & $0(0)$ & $0(0)$ & $0(0)$ & $1(100)$ & $1(0.99)$ \\
\hline Total & $10(20)$ & $40(80)$ & $9(17)$ & $44((83)$ & $103(100)$ \\
\hline
\end{tabular}

Note: $C O N s=$ coagulase-negative Staphylococcus, HAUTI = hospital-acquired UTI, CAUTI = community-acquired UTI.

was no association among other characteristics like sex, age, pregnancy, chronic disease, and recurrence of urinary tract infection $(P$ value $>0.05)$ (Table 4$)$.

The prevalence of bacteria significantly differs from inpatient 53/163(30.7\%) to outpatient 50/259 (19.3\%) UTIs. $(X 2=6.537, \quad \mathrm{OR}=1.753, \mathrm{CI}: 1.175-2.912, P=0.011) \quad(\mathrm{Ta}-$ ble 2). Those individuals who were inpatient were 1.753 times more likely exposed to develop HAUTIas compared to outpatient individuals. In addition, being female of sex was 8.925 times more likely to have increased urinary tract infection as compared with being male $(\mathrm{AOR}=8.925$; $\mathrm{CI}$ : $1.790-44.48, P=0.008$ ), and ages of $15-29$ years and $30-45$ years old were 0.126 and 0.057 less likely to have developed urinary tract infection when compared with ages of individuals whose age were $>60$ years old $(\mathrm{AOR}=0.126$; CI,0.020-0.792, $\quad P=0.027) \quad$ and $\quad(\mathrm{AOR}=0.057$; CI,0.057-0.480, $P=0.008$ ), respectively [Table 4].

In addition, individuals with the diabetic disease were 6.702 times more likely to have increased developing UTI as compared with individuals who were not diabetic $(\mathrm{AOR}=6.702$; $\mathrm{CI}, 1.994-22.528, P=0.002)$ [Table 4].
Besides these, previous usage of prolonged antibiotics was 5.689 times more likely to have developed urinary tract infection when compared with nonusers of antibiotics (AOR = 5.689; CI.1.840-17.590, $P=0.003$ ). Moreover, those patients who used catheters were 3.886 times more likely to have increased developing UTI as compared with those patients who have not used catheters $(\mathrm{AOR}=3.886$; CI,1.323-11.47, $P=0.014$ ) (Table 4).

However, there was no association among other characteristics like ages 0-14 years and 45-59 years, recurrent urinary tract infection, history of urinary tract obstruction, history of renal calculi, waiting for time in hospital, and duration of catheterization $(P$ value $>0.05)$ (Table 4$)$.

3.4. Antimicrobial Susceptibility of Community and HospitalAcquired Urinary Tract Infection. In this study, the highest degree of resistance among the 15 antimicrobial drugs was observed indifferent bacterial isolates. Klebsiella spp. was resistant to $100 \%$ for Ampicillin and $80 \%$ Augmentin in hospital-acquired and Tetracycline in community-acquired 


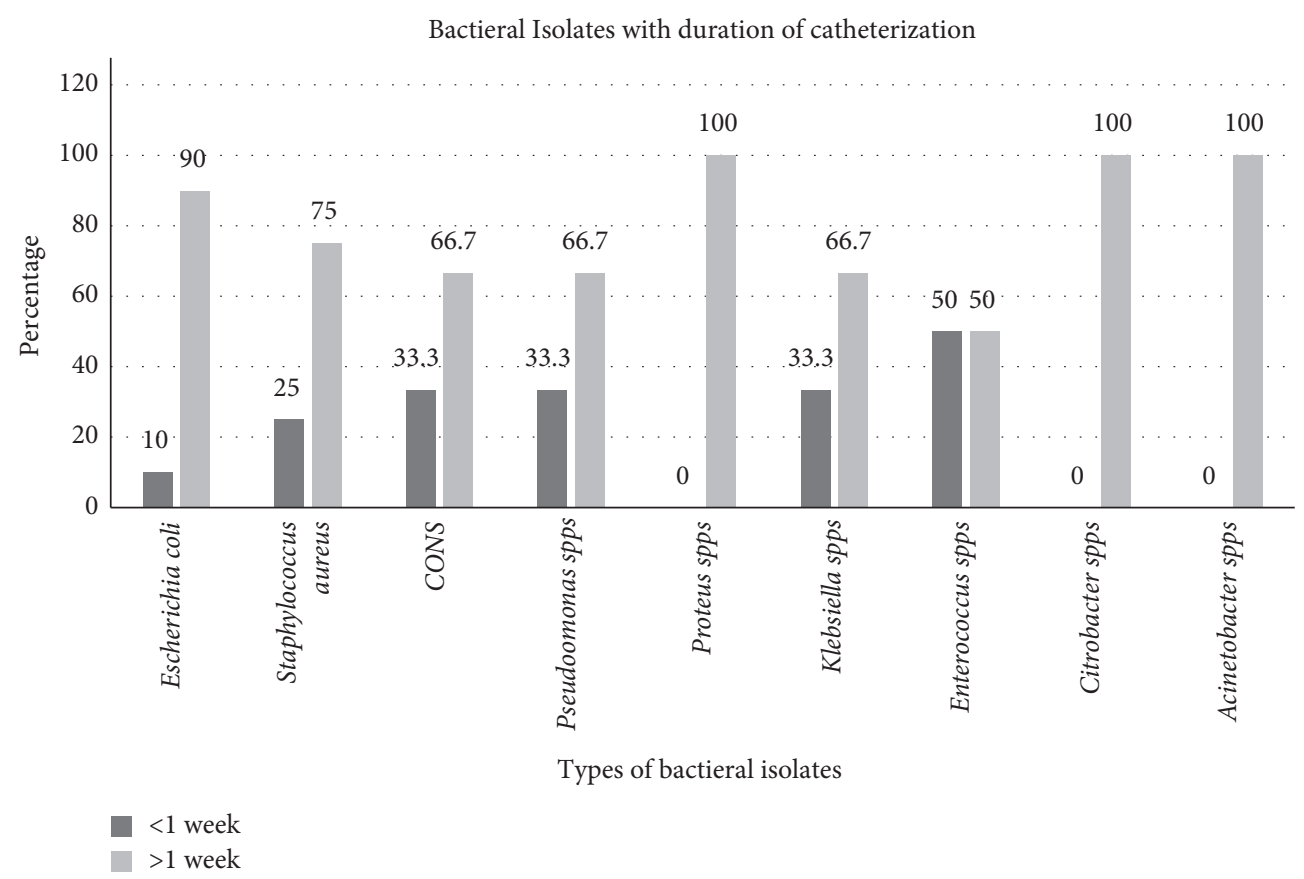

FIGURE 2: Distribution of isolates varies in the duration of catheterization at Dessie Referral Hospital.

TABLE 4: Bivariate and multivariate analyses of risk factors associated with community and hospital-acquired UTI among Dessie Referral Hospital.

\begin{tabular}{|c|c|c|c|c|c|c|c|c|}
\hline \multirow[b]{2}{*}{ Variable } & \multicolumn{4}{|c|}{ Hospital-acquired } & \multicolumn{4}{|c|}{ Community-acquired } \\
\hline & $\begin{array}{l}\text { Corollary }(95 \% \\
\text { CI })\end{array}$ & $P$ value & AOR $(95 \% \mathrm{CI})$ & $\begin{array}{c}P \\
\text { value }\end{array}$ & $\begin{array}{c}\text { Corollary }(95 \% \\
\text { CI })\end{array}$ & $P$ value & AOR $(95 \% \mathrm{CI})$ & $\begin{array}{c}P \\
\text { value }\end{array}$ \\
\hline \multicolumn{9}{|l|}{ Sex } \\
\hline Male & 1 & & & & 1 & & & \\
\hline Female & $3.01(1.336-6.803)$ & 0.008 & $8.93(1.79-44.5)$ & 0.008 & $2.3(1.10-4.94)$ & 0.026 & $0.4(0.072-2.06)$ & 0.266 \\
\hline \multicolumn{9}{|l|}{ Age } \\
\hline $0-14$ & $0.17(0.05-0.581)$ & 0.005 & $0.33(0.045-2.5)$ & 0.280 & $0.85(0.21-3.3)$ & 0.811 & & \\
\hline $15-29$ & $0.17(0.063-0.463)$ & $\leq 0.001$ & $0.13(0.02-0.79)$ & 0.027 & $2.8(0.86-9.13)$ & 0.085 & $3(0.476-19.24)$ & 0.240 \\
\hline $30-44$ & $0.06(0.018-0.218)$ & $\leq 0.001$ & $0.06(0.06-0.6)$ & 0.008 & $1.3(0.37-4.25)$ & 0.707 & & \\
\hline $45-59$ & $0.27(0.094-0.791)$ & 0.017 & $0.26(0.04-1.85)$ & 0.180 & $0.4(0.081-1.9)$ & 0.247 & & \\
\hline$>60$ & 1 & & & & 1 & & & \\
\hline \multicolumn{9}{|l|}{ Education } \\
\hline Illiterate & $1.29(0.291-5.766)$ & 0.733 & & & $1.5(0.44-4.82)$ & 0.553 & & \\
\hline Read and write only & $\begin{array}{c}1.23 \\
(0.287-5.233)\end{array}$ & 0.784 & & & $0.644(0.18-2.3)$ & 0.499 & & \\
\hline 1-8 grade complete & $0.60(0.129-2.794)$ & 0.515 & & & $0.92(0.26-3.26)$ & 0.893 & & \\
\hline $\begin{array}{l}\text { Up to grade } 12 \\
\text { complete }\end{array}$ & $1.17(0.133-10.22)$ & 0.889 & & & $1.63(0.3657 .36)$ & 0.521 & & \\
\hline University/college & 1 & & & & 1 & & & \\
\hline \multicolumn{9}{|l|}{ Residence } \\
\hline Urban & 1 & & & & 1 & & & \\
\hline Rural & $1.71(0.874-3.374)$ & 0.117 & $2.93(0.969-8.88)$ & 0.057 & $1.26(0.86-2.33)$ & 0.459 & & \\
\hline \multicolumn{9}{|l|}{ Monthly income } \\
\hline Lower $(<500 \mathrm{EBR})$ & $1.47(0.584-3.719)$ & 0.412 & & & $1.484(0.65-3.4)$ & 0.353 & & \\
\hline $\begin{array}{l}\text { Medium } \\
\text { (5001-1000EBR) }\end{array}$ & $0.61(0.203-1.821)$ & 0.374 & & & $1.1(0.449-2.75)$ & 0.820 & & \\
\hline Higher (>1001EBE) & 1 & & & & 1 & & & \\
\hline \multicolumn{9}{|l|}{ Pregnancy status } \\
\hline Yes & $2.39(0.54-10.612)$ & 0.252 & & & $3.72(1.1-12.53)$ & 0.034 & $\begin{array}{c}3.340 \\
(0.78-14.10)\end{array}$ & 0.102 \\
\hline No & 1 & & & & 1 & & & \\
\hline
\end{tabular}


TABLE 4: Continued.

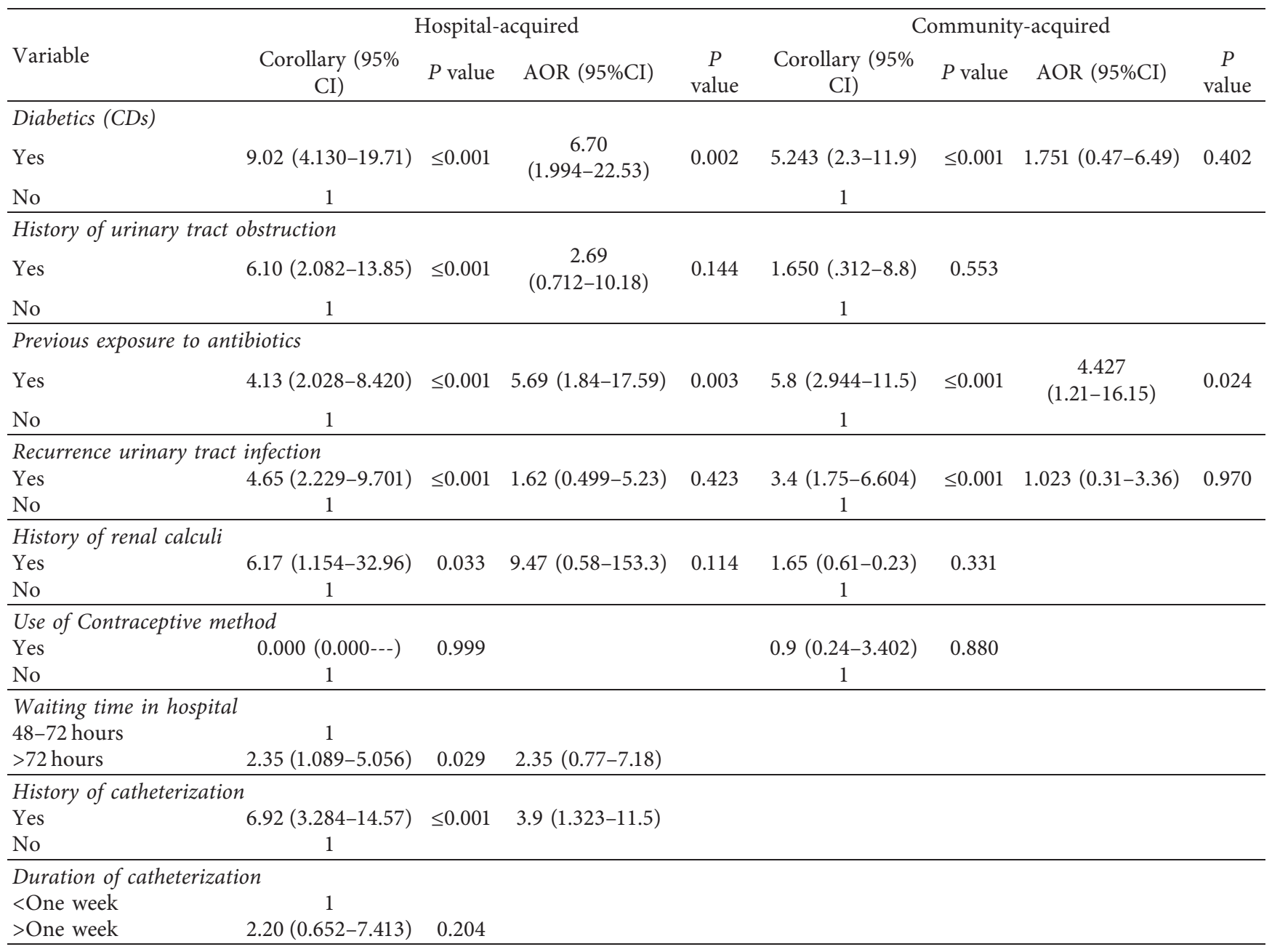

Note. COR: crude odd ratio; AOR: adjusted odd ratio; CI: confidence interval; EBR: Ethiopian birr; 1: reference.

UTI. Ampicillin, Tetracycline, and cotrimoxazole were resisted $100 \%$ by Proetus spp. in both study groups and Augmentin was $100 \%$ resisted in community-acquired UTI as well. Citrobacter spp. was $100 \%$ resistant to Augmentin, Tetracycline, Cotrimoxazole, and ceftriaxone. Acinetobacter spp. was $100 \%$ resistant to Tetracycline in hospital-acquired UTI. Penicillin and Ampicillin were $100 \%$ resisted by Enterococcus spp. in community-acquired urinary tract infections. (83.3\%) and (76.9\%) of E. coli isolates were resistant to Ampicillin in hospital and community-acquired urinary tract infections, respectively. $83.3 \%$ vs. $37.5,83.3$ vs. 37.5 , and $83.3 \%$ vs. $50 \%$ of CONs isolates in hospital and community-acquired urinary tract infection resist Penicillin, cotrimoxazole, and Tetracycline, respectively. $80 \%$ vs. $50 \%$ and $80 \%$ vs. $100 \%$ Augmentin and Tetracycline were resisted by Klebsiella spp. in hospital and community-acquired urinary tract infections, respectively (Table 5 and 6).

E. coli, Proetus spp., and Klebsiella spp. were susceptible to $100 \%$ for Meropenem in both community and hospitalacquired urinary tract infections, and Citrobacter spp., Pseudomonas spp., and Acinetobacter spp. were susceptible to $100 \%$ for Meropenem in hospital-acquired urinary tract infection. Even though the sample size is too small, Proteus spp., Klebsiella spp., Citrobacter spp., and Enterococcus spp. (\%100) were susceptible to Nitrofurantoin. Meropenem was the most sensitive drug $100 \%$ for both types of urinary tract infection (Table 5 and 6).

Multidrug resistance (MDR) was detected in 18 (69.2\%) and $11(61.1 \%)$ of E. coli isolates in both community and hospital-acquired urinary tract infections, respectively. The resistance patterns of 103 bacterial uropathogens isolated were tested against 15 antimicrobial agents. The predominant profile of E. coli MDR was observed in AMP, AMOXCLAV, TET, and SXT with 4 (3.85\%) among hospital-acquired and AMP, AUG, TET, CPR, SXT, and CTR with 2 (5.56\%) among community-acquired UTI. Among the total numbers, Proetus spp. isolates were 100\% MDR, three of them in hospital-acquired and the rest community-acquired. The scenario was also observed in Klebsiella spp. Moreover, S. aureus MDR was a higher proportion in hospital-acquired cases than community-acquired cases. In this study, antibiotic profile, except Penicillin, one $S$. aureus isolate resists all antimicrobials in hospital-acquired urinary tract infections (Table 7). 
TABLe 5: Antimicrobial susceptibility pattern of hospital-acquired urinary tract infection at Dessie Referral Hospital, Dessie.

\begin{tabular}{|c|c|c|c|c|c|c|c|c|c|c|c|c|}
\hline \multirow{2}{*}{$\begin{array}{l}\text { Isolates }(N=53) \\
\text { Gram-positive }\end{array}$} & \multicolumn{11}{|c|}{ Antimicrobial agent, $N(\%)$} & \\
\hline & & CIP & $\mathrm{TE}$ & $\mathrm{F}$ & SXT & $\mathrm{CN}$ & $P$ & AMP & VA & & & \\
\hline \multirow{3}{*}{ S. aureus $(n=13)$} & $\mathrm{S}$ & $9(69.2)$ & $2(15.4)$ & $9(60.2)$ & $5(38.5)$ & $10(77)$ & $4(33.3)$ & ND & ND & & & \\
\hline & $\mathrm{I}$ & $0(0)$ & $2(15.4)$ & $0(0)$ & $0(0)$ & $1(7.7)$ & $1(8.3)$ & & & & & \\
\hline & $\mathrm{R}$ & $4(30.8)$ & $\begin{array}{c}9 \\
(69.2) \\
\end{array}$ & $4(30.8)$ & $8(61.5)$ & $2(15.4)$ & $7(58.3)$ & & & & & \\
\hline \multirow{3}{*}{ CONS $(n=6)$} & $\mathrm{S}$ & $3(50)$ & $1(16.7)$ & $4(66.7)$ & $1(16.7)$ & $4(66.7)$ & $1(16.7)$ & ND & ND & & & \\
\hline & I & $1(16.7)$ & $0(0)$ & $0(0)$ & $0(0)$ & $0(0)$ & $0(0)$ & & & & & \\
\hline & $\mathrm{R}$ & $2(33.3)$ & $\begin{array}{c}5 \\
(83.3) \\
\end{array}$ & $2(33.3)$ & $5(83.3)$ & $2(23.3)$ & $5(83.3)$ & & & & & \\
\hline \multirow{3}{*}{$\begin{array}{l}\text { Enterococcus spp. } \\
(n=2)\end{array}$} & $\mathrm{S}$ & $1(50)$ & $0(0)$ & $2(100)$ & ND & ND & $1(50)$ & $1(50)$ & $2(100)$ & & & \\
\hline & $\mathrm{I}$ & $0(0)$ & $0(0)$ & $0(0)$ & & & $0(0)$ & $0(0)$ & $0(0)$ & & & \\
\hline & $\mathrm{R}$ & $1(50)$ & $2(100)$ & $0(0)$ & & & $1(50)$ & $1(50)$ & $0(0)$ & & & \\
\hline \multirow[t]{2}{*}{ Gram-negative } & & CIP & $\mathrm{T}$ & $\mathrm{F}$ & SXT & $\mathrm{CN}$ & AMP & MEM & $\mathrm{CRO}$ & NA & AMC & CAZ \\
\hline & $\mathrm{S}$ & $\begin{array}{c}12 \\
(66.7)\end{array}$ & $5(29)$ & $\begin{array}{c}16 \\
(88.9)\end{array}$ & $8(44.4)$ & $\begin{array}{c}12 \\
(66.7)\end{array}$ & $1(5.6)$ & $\begin{array}{c}18 \\
(100)\end{array}$ & $\begin{array}{c}15 \\
(83.3)\end{array}$ & $\begin{array}{c}15 \\
(83.3)\end{array}$ & $5(27.8)$ & $\begin{array}{c}12 \\
(66.7)\end{array}$ \\
\hline \multirow[t]{2}{*}{ E. $\operatorname{coli}(n=18)$} & I & $0(0)$ & $2(11)$ & $1(5.6)$ & $0(0)$ & $1(5.6)$ & $2(11.1)$ & $0(0)$ & $0(0)$ & $0(0)$ & $0(0)$ & $0(0)$ \\
\hline & $\mathrm{R}$ & $6(33.3)$ & $11(61)$ & $1(5.6)$ & $\begin{array}{c}10 \\
(55.6) \\
\end{array}$ & $5(27.7)$ & $\begin{array}{c}15 \\
(83.3) \\
\end{array}$ & $0(0)$ & $3(16.7)$ & $3(16.7)$ & $\begin{array}{c}13 \\
(72.2) \\
\end{array}$ & $6(33.3)$ \\
\hline \multirow{3}{*}{ Proteus spp. $(n=03)$} & $\mathrm{S}$ & $3(100)$ & $0(0)$ & $3(100)$ & $0(0)$ & $1(33.3)$ & $0(0)$ & $3(100)$ & $2(66.7)$ & $3(100)$ & $1(33.3)$ & $2(66.7)$ \\
\hline & I & $0(0)$ & $0(0)$ & $0(0)$ & $0(0)$ & $0(0)$ & $0(0)$ & $0(0)$ & $0(0)$ & $0(0)$ & $0(0)$ & $0(0)$ \\
\hline & $\mathrm{R}$ & $0(0)$ & $3(100)$ & $0(0)$ & $3(100)$ & $2(66.7)$ & $3(100)$ & $0(0)$ & $1(33.3)$ & $0(0)$ & $2(66.7)$ & $1(33.3)$ \\
\hline \multirow{3}{*}{ Klebseilla spp. $(n=5)$} & $S$ & $3(60)$ & $0(0)$ & $5(100)$ & $2(40)$ & $3(60)$ & $0(0)$ & $5(100)$ & $2(40)$ & $4(80)$ & $0(0)$ & $4(80)$ \\
\hline & I & $1(20)$ & $1(20)$ & $0(0)$ & $0(0)$ & $0(0)$ & $0(0)$ & $0(0)$ & $0(0)$ & $1(20)$ & $1(20)$ & $0(0)$ \\
\hline & $\mathrm{R}$ & $1(20)$ & $4(80)$ & $0(0)$ & $3(60)$ & $2(30)$ & $5(100)$ & $0(0)$ & $3(60)$ & $0(0)$ & $4(80)$ & $1(20)$ \\
\hline \multirow{3}{*}{$\begin{array}{l}\text { Citrobacter spp. } \\
(n=2)\end{array}$} & $S$ & $1(50)$ & $0(0)$ & $2(100)$ & $0(0)$ & $1(50)$ & $0(0)$ & $2(100)$ & $0(0)$ & $1(50)$ & $0(0)$ & $1(50)$ \\
\hline & I & $1(50)$ & $0(0)$ & $0(0)$ & $0(0)$ & $1(50)$ & $0(0)$ & $0(0)$ & $0(0)$ & $1(50)$ & $0(0)$ & $0(0)$ \\
\hline & $\mathrm{R}$ & $0(0)$ & $2(100)$ & $0(0)$ & $2(100)$ & $0(0)$ & $2(100)$ & $0(0)$ & $2(100)$ & $0(0)$ & $2(100)$ & $1(50)$ \\
\hline \multirow{3}{*}{$\begin{array}{l}\text { Pseudomonas spp. } \\
(n=3\end{array}$} & $\mathrm{S}$ & $0(0)$ & ND & ND & ND & $2(66.7)$ & ND & $3(100)$ & ND & ND & ND & ND \\
\hline & I & $1(33.3)$ & & & & $0(0)$ & & $0(0)$ & & & & \\
\hline & $\mathrm{R}$ & $2(66.7)$ & & & & $1(33.3)$ & & $0(0)$ & & & & \\
\hline \multirow{3}{*}{$\begin{array}{l}\text { Acetobacter spp. } \\
(n=1)\end{array}$} & $\mathrm{S}$ & $1(100)$ & $0(0)$ & ND & $0(0)$ & $0(0)$ & ND & $1(100)$ & ND & ND & ND & ND \\
\hline & I & $0(0)$ & $1(100)$ & & $0(0)$ & $1(100)$ & & $0(0)$ & & & & \\
\hline & $\mathrm{R}$ & $0(0)$ & $0(0)$ & & $1(100)$ & $0(0)$ & & $0(0)$ & & & & \\
\hline \multirow{3}{*}{ Total } & S & $\begin{array}{c}33 \\
(37.4)\end{array}$ & $8(16)$ & $\begin{array}{c}41 \\
(83.6)\end{array}$ & $15(33)$ & $\begin{array}{c}33 \\
(64.7)\end{array}$ & 7 (15) & 33 (97) & $21(70)$ & $23(82)$ & $6(21)$ & 19 (68) \\
\hline & I & $5(9.4)$ & $6(4)$ & $1(2)$ & $0(0)$ & $4(7.8)$ & $3(6)$ & $0(0)$ & $0(0)$ & $2(7)$ & $1(4)$ & $0(0)$ \\
\hline & $\mathrm{R}$ & $15(28)$ & $36(80)$ & 7 (14.4) & $32(67)$ & $\begin{array}{c}14 \\
(27.4)\end{array}$ & $38(79)$ & $1(3)$ & $9(30)$ & $3(11)$ & $21(75)$ & $9(32)$ \\
\hline
\end{tabular}

Note: AMP, ampicillin; CIP, ciprofloxacin; CRO, ceftriaxone; AMC, amoxicillin-clavulanate; CN, gentamicin; F, nitrofurantoin; SXT, cotrimoxazole; NA, nalidixic acid; CAZ, ceftazidime; MEM, meropenem; VA, vancomycin; TE, tetracycline; ND, not done.

\section{Discussion}

Urinary tract infection is the most common infectious disease in both community and hospital-acquired settings. In this study, the overall prevalence of urinary tract infection was $23.7 \%$ (95\%CI: 19.3-27.5), which lies between the low prevalence of (8.7\%) in Iran [27], and the high prevalence of $90.1 \%$ Ethiopia-Shashemenie [28], in the different areas of the world. The results of this study also showed that the etiologic agents of UTIs mainly belonged more Gramnegative bacilli 61/103 (59.22\%) than Gram-positive cocci $42 / 103(40.78 \%)$. It is a known fact that Gram-negative isolates are the most prominent uropathogens compared to Gram-positive isolates, and the common source of pathogens causing UTI is intestinal flora which contains many
Gram-negative organisms. Hence, the infection may be due to fecal contamination arising from poor hygiene [29].

The present study showed that the prevalence of CAUTI and HAUTI was 50 (19.7\%) (95\%CI:16.0-24.7) and 50 (30.7\%) (95\%CI:23.3-38), respectively. This prevalence was comparable with Nigeria, (36\%) HAUTI [5] and Israel, $24.2 \%$ CAUTI [30]. But, lower than a study done in Northwestern India (44.27\% vss 39.8\%) [31], Saudi Arabia (55.3\% vs. $44.7 \%$ [32], Bangladesh (45\% vs. 50\%) [33], Kuwait (59\% vs. 41\%) [34], Congo (35\% vs. 65\%) [21], and Yemen(51.2 vs. 48.8) [34] CAUTI and HAUTI, respectively, and higher than studies done in Nigeria (14.9\% vs. $11.1 \%)$ [35], Southwestern Nigeria CAUTI (11\%) [36], and Bahir Dar (9.4\%) HAUTI (37). The reality might justify the variation that differences exist in the sample size of study 
TABLe 6: Antimicrobial susceptibility pattern of community-acquired urinary tract infection at Dessie Referral Hospital, Dessie.

\begin{tabular}{|c|c|c|c|c|c|c|c|c|c|c|c|c|}
\hline \multirow{2}{*}{$\begin{array}{l}\text { Isolates }(N=50) \\
\text { Gram-positive }\end{array}$} & \multicolumn{11}{|c|}{ Antimicrobial agent, $N(\%)$} & \\
\hline & & CIP & $\mathrm{TE}$ & $\mathrm{F}$ & SXT & $\mathrm{CN}$ & $P$ & AMP & VA & & & \\
\hline \multirow{3}{*}{ S. aureus $(n=12)$} & $S$ & $8(66.7)$ & $1(8.3)$ & $12(100)$ & $5(41.7)$ & $9(75)$ & $7(58.3)$ & ND & ND & & & \\
\hline & I & $0(0)$ & $3(25)$ & $0(0)$ & $1(8.3)$ & $0(0)$ & $1(8.3)$ & & & & & \\
\hline & $\mathrm{R}$ & $4(33.3)$ & $8(66.7)$ & $0(0)$ & $6(8.3)$ & $3(25)$ & $4(33.3)$ & & & & & \\
\hline \multirow{3}{*}{ CONS $(n=8)$} & $S$ & $6(75)$ & $3(37.5)$ & $7(87)$ & $5(62.5)$ & $7(87.5)$ & $5(62.5)$ & ND & ND & & & \\
\hline & $\mathrm{I}$ & $1(12.5)$ & $1(12.5)$ & $1(12.5)$ & $0(0)$ & $0(0)$ & $0(0)$ & & & & & \\
\hline & $\mathrm{R}$ & $1(12)$ & $4(50)$ & $0(0)$ & $3(37.5)$ & $1(12.5)$ & $3(37.5)$ & & & & & \\
\hline \multirow{3}{*}{$\begin{array}{l}\text { Enterococcus spp. } \\
(n=1)\end{array}$} & $\mathrm{S}$ & $1(100)$ & $0(0)$ & $1(100)$ & ND & ND & $0(0)$ & $0(0)$ & $0(0)$ & & & \\
\hline & $\mathrm{I}$ & $0(0)$ & $0(0)$ & $0(0)$ & & & $0(0)$ & $0(0)$ & $1(100)$ & & & \\
\hline & $\mathrm{R}$ & $0(0)$ & $1(100)$ & $0(0)$ & & & $1(100)$ & $1(100)$ & $0(0)$ & & & \\
\hline \multirow[t]{2}{*}{ Gram-negative } & & CIP & $\mathrm{T}$ & $\mathrm{F}$ & SXT & $\mathrm{CN}$ & AMP & MEM & $\mathrm{CRO}$ & NA & $\mathrm{AMC}$ & CAZ \\
\hline & $\mathrm{S}$ & 24 & $9(34.5)$ & 25 & 12 & 21 & $5(19.2)$ & 26 & 21 & 18 & 11 & 22 \\
\hline \multirow{2}{*}{ E. $\operatorname{coli}(n=26)$} & I & $\begin{array}{c}(92.3) \\
1(3.8)\end{array}$ & $0(0)$ & $\begin{array}{l}(96.2) \\
1(3.8)\end{array}$ & $\begin{array}{c}(46.2) \\
0(0)\end{array}$ & $\begin{array}{c}(80.8) \\
1(3.8)\end{array}$ & $1(3.8)$ & $\begin{array}{l}(100) \\
0(0)\end{array}$ & $\begin{array}{c}(80.8) \\
2(7.7)\end{array}$ & $\begin{array}{c}(69.2) \\
0(0)\end{array}$ & $\begin{array}{c}(42.3) \\
1(3.8)\end{array}$ & $\begin{array}{c}(84.6) \\
2(3.8)\end{array}$ \\
\hline & $\mathrm{R}$ & $1(3.8)$ & $\begin{array}{c}17 \\
(65.4)\end{array}$ & $0(0)$ & $\begin{array}{c}14 \\
(53.8)\end{array}$ & $4(15.4)$ & $\begin{array}{c}20 \\
(76.9)\end{array}$ & $0(0)$ & $3(11.5)$ & $8(30.8)$ & $\begin{array}{c}14 \\
(53.8)\end{array}$ & $3(11.5)$ \\
\hline \multirow{3}{*}{ Proteus spp. $(n=1)$} & $S$ & $1(100)$ & $0(0)$ & $1(100)$ & $0(0)$ & $0(0$ & $0(0)$ & $1(100)$ & $1(100)$ & $1(100)$ & $0(0)$ & $1(100)$ \\
\hline & I & $0(0)$ & $0(0)$ & $0(0)$ & $0(0)$ & $1(100)$ & $0(0)$ & $0(0)$ & $0(0)$ & $0(0)$ & $0(0)$ & $0(0)$ \\
\hline & $\mathrm{R}$ & $0(0)$ & $1(100)$ & $0(0)$ & $1(100)$ & $0(0)$ & $1(100)$ & $0(0)$ & $0(0)$ & $0(0)$ & $1(100)$ & $0(0)$ \\
\hline \multirow{3}{*}{ Klebsiella spp. $(n=2)$} & $\mathrm{S}$ & $1(50)$ & $0(0)$ & $2(100)$ & $1(50)$ & $1(50)$ & $0(0)$ & $1(100)$ & $1(50)$ & $1(50)$ & $1(50)$ & $1(50)$ \\
\hline & I & $0(0)$ & $0(0)$ & $0(0)$ & $0(0)$ & $0(0)$ & $0(0)$ & $0(0)$ & $0(0)$ & $0(0)$ & $0(0)$ & $0(0)$ \\
\hline & $\mathrm{R}$ & $1(50)$ & $2(100)$ & $0(0)$ & $1(50)$ & $1(50)$ & $2(100)$ & $0(0)$ & $1(50)$ & $1(50)$ & $1(50)$ & $1(50)$ \\
\hline \multirow{3}{*}{ Total } & $\mathrm{S}$ & $41(82)$ & $13(26)$ & $48(96)$ & $23(48)$ & $38(76)$ & $18(36)$ & $28(93)$ & $23(77)$ & $20(69)$ & $\begin{array}{c}12 \\
(41.4)\end{array}$ & $24(80)$ \\
\hline & I & $2(4)$ & $4(8)$ & $2(4)$ & $1(2)$ & $2(4)$ & $2(4)$ & $0(0)$ & $3(10)$ & $0(0)$ & $1(3.4)$ & $2(7)$ \\
\hline & $\mathrm{R}$ & $7(14)$ & $33(66)$ & $0(0)$ & $25(50)$ & $9(20)$ & $30(60)$ & $1(7)$ & $4(13)$ & $9(31)$ & $16(55)$ & $4(13)$ \\
\hline
\end{tabular}

Note: AMP, ampicillin; CIP, ciprofloxacin; CRO, ceftriaxone; AMC, amoxicillin-clavulanate; CN, gentamicin; F, Nitrofurantoin; SXT, cotrimoxazole; NA, nalidixic acid; CAZ, ceftazidime; MEM, meropenem; VA, vancomycin; TE, tetracycline; ND, not done.

participants, time of study period, study setting (community-based or hospital-based), characteristics of the population studied (age, residence, immunological status, urological disorders, socioeconomic status, educational level, hygiene practice), and laboratory methods.

According to our study, E. coli was the predominant uropathogen and no significant change has occurred in terms of pattern or position, of 26/50 (52\%) and 18/53 (33.96\%) in community and hospital-acquired UTI, respectively. This report is comparable with Nigeria [5], Saudi Arabia(38), and Kuwait [33]. It might be due to several virulent factors specific for colonization and invasion of the urinary epithelium, such as P-fimbria and S-fimbria adhesion. However, the frequency of isolation of $E$. coli in urine samples varies in different study areas. It may be due to the high variation of different species of bacteria in the study and differ in the laboratory method of isolation. This makes it difficult to compare.

The frequency of CAUTI caused by E. coli is higher than that of HAUTI in this study. This is because most of the bacterial organisms causing UTI originate from the fecal flora and are dominated by various virulence factors that facilitate the ascent of bacteria from the perianal area to the urethra into the bladder and less frequently allow the organisms to reach the kidneys to induce symptomatic inflammation [38].

The second most frequent bacteria isolated were S. aureus at isolation rates of ( $24 \%$ vs. $24.5 \%)$ in CAUT and
HAUTI, respectively. This result was a similar pattern with the study in Arbaminch, Ethiopia [29], and Southwestern Nigeria [39] in CAUTI and HAUTI, respectively. This study was at variance with other studies that reported a higher prevalence of other Gram-negative enteric bacilli Congo [21] K. pneumoniae, Saudi Arabia [32] Enterobacter spp., and Bangladesh [33] Pseudomonas spp. compared to as S. aureus.

In our study, coagulase-negative staphylococcus was the third position 8/50(16\%) and 6/53 (11.3\%) for CAUTI and HAUTI, respectively. However, our result was not correlated with other reports in Saudi Arabia [32], Bangladesh [33], and Abuja Nigeria [5] Pseudomonas spp. and is a retrospective study in Dessie regional lab, the second and third isolates were Klebsiella spp. and Proteus spp., respectively [40]. The possible reason for this variance was most Gram-positive bacteria survived commensally and it has been shifting with the environmental conditions such as temperature, humidity and resistance patterns. On the other hand, an increase in Staphylococcal UTI in the hospital setup may increase the use of instrumentation such as a catheter [41].

In our study, the prevalence of Klebsiella spp. was higher in hospital-acquired setting 5/53 (9.4\%) than in community-acquired setting $2 / 50(4 \%)$. In this study, it can be justified by its ability for adaptation to the hospital environment, and it can survive longer than other bacteria, enabling cross-infection within hospitals [42]. This report is correlated with the study in Bangladesh [33]. Citrobacter spp., Pseudomonas spp., and Acinetobacter spp. were 
TABLE 7: Multidrug resistance pattern of community and hospital-acquired bacterial isolates among Dessie Referral Hospital UTI suspected patients at Dessie, March-April, 2019.

\begin{tabular}{|c|c|c|c|c|}
\hline \multirow{2}{*}{ Isolates } & \multicolumn{2}{|l|}{ Community-acquired isolates } & \multicolumn{2}{|l|}{ Hospital-acquired isolates } \\
\hline & Antimicrobial agents & $\mathrm{N}(\%)$ & Antimicrobial agents & $N(\%)$ \\
\hline \multirow[t]{6}{*}{ E. coli [18]-IPD } & AMC, SXT, CXM & $1(3.85)$ & AMP, AMOX-CLA, GEN & $1(5.56)$ \\
\hline & AMP, AMOX-CLAV, SXT, CTR & $1(3.85)$ & AMP, AMOX-CLAV, TET, SXT, CXM & $1(5.56$ \\
\hline & NAL, SXT, CTR & $1(3.85)$ & AMOX-CLAV, SXT, CXM & $1(5.56)$ \\
\hline & AMP, AMOX-CLAV, TET & $2(3.85)$ & AMP, AMOX-CLAV, TET CPRSXT, CXM & $1(5.56)$ \\
\hline & AMP, AMOX-CLAV, TET, SXT & $4(3.85)$ & AMP AMOX-CLAV CPR GEN, NIT & $1(5.56)$ \\
\hline & TET, SXT, GEN & $1(3.85)$ & AMP, TET, CPR & $1(5.56)$ \\
\hline \multirow{7}{*}{ E. coli $[26]-\mathrm{OPD}$} & AMP, AMOX-CLAV NAL, TET, GEN & $1(3.85)$ & AMP, NAL, TET & $1(5.56)$ \\
\hline & AMP, NAL, TET & $1(3.85)$ & AMP, TET, CPR, SXT, CTR & $2(5.56)$ \\
\hline & AMP, AMOX-CLA, NAL TET, SXT & $2(3.85)$ & AMP, AMOX-CLAV, CPR & $1(5.56)$ \\
\hline & AMP, AMOX-CLAV NAL, SXT & $1(3.85)$ & AMP AMOX-CLAV, SXT & $1(5.56)$ \\
\hline & AMP, TET, SXT & $1(3.85)$ & & \\
\hline & AMP, TET, SXT, GEN & $1(3.85)$ & & \\
\hline & AMOX-CLAV, SXT & $1(3.85)$ & & \\
\hline Total & & $18(69.2)$ & & $11(61.1)$ \\
\hline Proetus spp. [3]-IPD & & & AMP, TET, SXT, GEN & $1(33.3)$ \\
\hline \multirow{2}{*}{ Proteus spp. (1)-OPD } & AMP, AMOX-CLAV, TET, SXT & $1(100)$ & AMP, AMOX-CLAV, TET SXT & $1(33.33)$ \\
\hline & & & AMP, AMOX-CLAV, TET, SXT, GEN, CTR & $1(33.33)$ \\
\hline Total & & $1(100)$ & & $3(100)$ \\
\hline \multirow[t]{2}{*}{ Klebsiella spp. [5]-IPD } & AMP, NAL, TET, SXT, GEN, CTR & $1(50)$ & AMP, AMOX-CLAV, TET & $1(20)$ \\
\hline & & & AMP, AMOX-CLV SXT, GEN, CTR & $1(20)$ \\
\hline \multirow{3}{*}{ Klebsiella spp. [2]- OPD } & AMP AMOX_CI AV NAI TFT CPR & $1(50)$ & AMP, AMOX-CLAV, TET, CTR & $1(20)$ \\
\hline & AIVI, AIVIUA-CLAV NAL, IEI, CPK & $1(30)$ & AMP, NAL, TET, CPR, SXT, CTR & $1(20)$ \\
\hline & & & AMP, AMOX-CLAV, SXT & $1(20)$ \\
\hline Total & & $2(100)$ & & $5(100)$ \\
\hline \multirow{2}{*}{ Citrobacter spp [2] } & & & AMOX-CLAV, AMP, NAL, SXT, GEN, CTR & $1(50)$ \\
\hline & & & AMP, AMOX-CLAV, TET, SXT, CTR & $1(50)$ \\
\hline Total & & & & $2(100)$ \\
\hline \multirow[t]{3}{*}{ S. aureus [13]-IPD } & TET, CPR, PEN, GEN & $1(8.3)$ & TET, SXT, PEN & $3(7.7)$ \\
\hline & & & TET, CPR, SXT, GEN, NIT & $1(7.7)$ \\
\hline & & & TET, CPR, SXT, PEN & $1(7.7)$ \\
\hline \multirow[t]{3}{*}{ S. aureus [12]-OPD } & TET, CPR, SXT, PEN & $1(8.3)$ & CPR, SXT, PEN & $1(7.7)$ \\
\hline & & & TET, PEN, NIT & $1(7.7)$ \\
\hline & & & & $7(53.9)$ \\
\hline Total & & $2(16.6)$ & & \\
\hline CONS [6]-IPD & TET, CPR, SXT & $1(12.5)$ & TET, PEN, GEN, CPR & $1(16.6)$ \\
\hline \multirow[t]{3}{*}{ CONS [8]-OPD } & TET, PEN, GEN & $1(12.5)$ & TET, SXT, PEN & $1(16.6)$ \\
\hline & & & TET SXT, GEN & $1(16.6)$ \\
\hline & & & TET, CPR, SXT, PEN, GEN & $1(16.6)$ \\
\hline Total & & $2(25)$ & & $4(66.7)$ \\
\hline
\end{tabular}

Note: $\mathrm{IPD}=$ inpatient, $\mathrm{OPD}=$ outpatient department, $\mathrm{CONs}=$ coagulase-negative Staphylococcus, $\mathrm{AMP}=$ ampicillin, $\mathrm{TET}=$ tetracycline, $\mathrm{PEN}=$ penicillin, $\mathrm{GEN}=$ gentamycin, $\mathrm{CPR}=$ ciprofloxacillin, $\mathrm{SXT}=$ sulphamethoxazole-trimethoprim, NIT = nitrofurantoin, $\mathrm{CTR}=$ ceftriaxone, $\mathrm{CXM}=$ cefixime .

isolated from hospitalized patients only. Similar results were found in Yemen [34]. Pseudomonas spp. is enabled to survive and thrive well in soaps and disinfectants used for urethral catheterization [35]. Antimicrobial resistance has been recognized as an emerging worldwide problem in both ideveloped and developing countries. In hospital-acquired urinary tract infections, the resistance rate of Gram-negative isolates was $86.6 \%$ and $75 \%$ for Ampicillin and Amoxclav, respectively. In Gram-positive isolates, $76 \%$ and $68.4 \%$ were resistant to Tetracycline and SXT, respectively, except Enterococcus spp. This result was comparable with Yemen [37].
In community-acquired urinary tract infections, resistance for Ampicillin and Tetracycline was $79.3 \%$ and $68.9 \%$ to Gram-negative isolates and $61.9 \%$ and $36 \%$ for Tetracycline and SXT to Gram-positive isolates, respectively. This report is in agreement with Jordan (73\%) [43] and Yemen (tetracycline (68.7\%)) [34].The high proportion of resistance found in ampicillin, tetracycline, amox-clav, and SXT in both settings could be explained by the long period for which these drugs have been available and in use for UTI and in our study area, people having easy access to antibiotics in drug shops and therefore greater intake that contributes to increased the proportion of resistance. 
However, meropenem and nitrofurantoin were the most active drugs for both types of UTI because they are not easily accessible. This report was similar to Northwestern India for Nitrofurantoin [31].

In this study, the overall prevalence of multidrug resistance was $55.3 \%$ (57/103) (95\%CI:10.0-16.8), in which $72.2 \%(21 / 29)$ of Gram-negative and 20\% (4/20) of Grampositive isolates in CAUTI and 75\% (21/28) of Gram-negative and 57\% (11/19) of Gram-positive isolates in HAUTI were observed; this finding showed that Gram-negative isolates were almost equally distributed. In both settings, this may be due to multidrug resistance bacteria circulating from hospital setting to community setting and vice versa. On the other hand, almost twofold Gram-positive MDR isolates were seen in hospital setup; most probably due to instrumentation or unsafe healthcare practice. This report was lower than the study in Gondar [44]. The possible reason for this result was geographical variation. Different demographic characteristics in various studies have been described to be associated with an increase in community and hospital-acquired UTI. In our study on CAUTI, previous use of antibiotics was significantly associated $(P=0.024)$ with the prevalence of CAUTI. Of $39.4 \%$ of the study subjects who had previously used antibiotics for UTI or other than UTI, $36.3 \%$ were culture-positive. This finding was consistent with other studies, India [45] in Gondar, Ethiopia [46]. The possible reason for this finding is that the common source of pathogens causing UTI is intestinal flora exposed to too many antibiotic classes for UTI and other than UTI bacterial diseases, and hence damaging intestinal flora, then allowing uropathogens to colonize and subsequently infect the urinary tract [47]. In the previous retrospective study, Dessie regional lab, being female, and age were risk factors of developing CAUTI as compared to males. But in this study, none of them were associated with these infections. The differences observed in this study might be because of characteristics of the population studied (immunological status, urological disorder), and most isolates from the community that is tested in the regional lab may be predominantly from referral patients for whose previous antimicrobial treatment failed.

In HAUTI, patient setting, sex, age, diabetic mellitus, catheterization, and previous use of antibiotics have a statistically significant relationship with significant bacteriuria. Similar studies were found in Gondar-Ethiopia [46], HararEthiopia [36], and Uganda [48]. The high prevalence of bacteriuria among inpatients 53/103(51.5\%) as compared to the outpatients, 50/103 (48.5\%) was that increased risk of infection due to indwelling catheter that contributed to $64.4 \%$ of the inpatients UTIs. $(X 2=6.537, P=0.011)$. This study is comparable with the study conducted in Uganda (49) and India [45] because infections could have been acquired through unsafe healthcare practices such as catheterization.

In the present study, the difference in the incidence of HAUTI among the males was $16.7 \%$ (9/54), and females $37.6 \%(41 / 109)$ was statically significant $(P=0.008)$. This indicates that females have stronger predictions for HAUTI compared to males. The possible reason for this finding is females are more catheterized than males due to obstetric and gynecological causes (urinary tract abnormalities or obstruction) and shorter length of female urethra, its proximity to the anal canal, and absence of prostatic secretions. This report is similar to the study in Bangladesh [33].

In this study, the age range of $15-29$ years, the isolation rate of CAUTI and HAUTI were $91 \%$ in females and $8.7 \%$ in males and $0 \%$ male, and $100 \%$ in females, respectively. This high frequency is due to the sexually active stage in females or probably pregnant women. On the other hand, old age (>60 years) $50 \%$ female and $50 \%$ male and $29.2 \%$ in male and $70.8 \%$ in female in CAUTI and HAUTI were found, respectively. The high isolation rate of UTI among the old age group could be due to Genito-urinary atrophy and vaginal prolapse after menopause which increasing vaginal $\mathrm{pH}$ and decreasing vaginal Lactobacillus that allows Gram-negative bacteria to grow and act asuropathogens [33]. Moreover, it is the possible reason for males' prostatic gland enlargement and decrease of bacteriostatic prostatic secretions might account for such infections [33]. This finding is comparable with Bangladesh [33], Nigeria [49], and Shashemenie in Ethiopia [28].

In hospital-acquired urinary tract infections, previous use of antibiotics was significantly associated $(P=0.003)$ with the prevalence of hospital-acquired urinary tract infections. Of the total of $31.9 \%$ of previous antibiotic users for UTI or other than UTI, 52\% of them were culture-positive. Our studies reflected that the prior and continuous use of antibiotics correlates with the UTI because the widespread use of antibiotics may cause multiple drug resistance microorganisms, this finding correlated with the study conducted in India [50] and Bangladesh [33].

In this study, urinary catheterization was the leading one among the causes of UTI due to instrumentation. From this study, $31.2 \%$ of UTI symptomatic study subjects used catheters in the hospital settings, $64.4 \%$ of those study participants were culture-positive. This reflects the greater proportion of HAUTI was significantly associated $(P=0.014)$ with catheterization. This finding is comparable with the study in Bangladesh [33], India [45], and Bahir DarEthiopia [48].

The statistically significant association between HAUTI and diabetes $(P=0.002)$ could be due to altered immunity in diabetic patients, which includes depressed polymorphonuclear leukocyte functions, altered leukocyte adherence, chemotaxis, phagocytosis, the impaired bactericidal activity of the antioxidant system [51], and neuropathic complications, such as impaired bladder emptying. Moreover, a higher glucose concentration in the urine may create a culture medium for pathogenic microorganisms in diabetic patients that may result in this UTI. Similar reports are shown in Harar-Ethiopia [36], Nepal [52] India [45], and Uganda [48].

\section{Conclusion and Recommendations}

Findings of this study revealed that the overall prevalence rate of UTI was slightly high, and the hospital-acquired UTI 
group of patients has a higher rate of infection than community-acquired infection. E. coli is still the leading cause of community and hospital-acquired UTI, along with its increasing resistance pattern to different antibiotics, and is going to be an alarming health hazard. This study has shown that the alarming level of resistance (Ampicillin) achieved by bacteria is involved in causing UTI. E. coli and various isolates were more sensitive to meropenem and nitrofurantoin compared to other antibiotics tested.

The healthcare policy should be discouraging inappropriate use of antibiotics and prevent further development of resistant strains among bacteria. A continual audit of antimicrobial susceptibility patterns among the community and hospital-acquired UTI as a cause of morbidity should be performed and the findings should be reviewed periodically. Awareness should be created among the community members to prevent risk factors associated with the infection. Nitrofurantoin should be the first choice for empirical treatment of UTI in this study area. Further research should be focused on the effectiveness of risk factor reducing strategies and the changes to economic costs and healthcare benefits.

\section{Limitation of the Study}

This study did not consider anaerobic bacteria and few bacterial isolates were not identified at the species level that causes UTI due to lack of facility. Our study limitation was using only the antibiotics disks diffusion method to perform antimicrobial susceptibility instead of the microdilution method.

\section{Abbreviations}

ATCC: American Type Culture Collection

CAUTI: Community-acquired urinary tract infection

CFU: Colony forming unit

CLSI: Clinical and Laboratory Standard Institute

CONS: Coagulase-negative Staphylococci

HAUTI: Hospital-acquired urinary tract infection

HCWs: Healthcare workers

HPF: High power field

IPD: Inpatient department

OPD: Outpatient department

SOP: Standard operating procedure

SPSS: $\quad$ Statistical Package for Social Sciences

UTI: Urinary tract infection

WHO: World Health Organization.

\section{Data Availability}

Data used to support the finding of this study are available from the corresponding author upon request.

\section{Ethical Approval}

Ethical clearance was obtained from the School of Biomedical and Laboratory Sciences, University of Gondar ethical review committee, and a letter informing Dessie Referral Hospital Dessie about the purpose of the study and permission was obtained from Dessie Referral Hospital to access data from the study population.

\section{Consent}

Consent was obtained from study participants after explaining the purpose and objective of the study. If study participants are children (under 16 years of age), consent to participate was collected from the parents/guardians in written form. Study participants who were not willing to participate in the study were not forced to participate. Study participants were informed that all data and samples obtained from them were kept confidential by using codes instead of any personal identifiers and are meant only for the study. For those study participants who were positive, we advised going to the health institution to consult the clinicians for medical treatment and treated with the respective antibiotics;

\section{Conflicts of Interest}

The authors declare that they have no conflicts of interest.

\section{Authors' Contributions}

BA conceptualized the study, contributed to the format of the data collection instruments, contributed to the study protocol, and developed the data collection tools for field data collection and analysis. MJ and BS participated in the pretest, supervised the actual data collection process, entered the data, and significantly contributed to the analysis part. All authors participated in the data analysis, interpretation, and development of the manuscript. All authors read and approved the final manuscript.

\section{Acknowledgments}

The authors would like to acknowledge University of Gondar, Department of Medical Laboratory Sciences, Amhara Regional Health Bureau. Furthermore, all study participants, data collectors, and Amhara Public Health Institute and Dessie Referral Hospital are highly acknowledged. This study was fully granted by University of Gondar.

\section{References}

[1] A. Chaturvedi, R. Garg, and V. A. Singh, "Comparative evaluation of different culture media for the isolation and identification of common urinary pathogens," International Journal of Research in Medical Sciences, vol. 5, no. 8, pp. 3676-3679, 2017.

[2] F. D. Otajevwo, "Urinary tract infection among symptomatic outpatients visiting a tertiary hospital based in midwestern Nigeria," Global Journal of Health Science, vol. 5, no. 2, pp. 187-99, 2013.

[3] L. E. Nicolle, "Urinary tract infection," Nephrology Secrets, vol. 18, pp. 340-345, 2012.

[4] M. Arjunan, A. A. Al-Salamah, and M. Amuthan, "Prevalence and antibiotics susceptibility of uropathogens in patients from a rural environment, Tamilnadu," American Journal of Infectious Diseases, vol. 6, no. 2, pp. 29-33, 2010. 
[5] V. Pai and B. Nair, "Etiology and sensitivity of uropathogens in outpatients and inpatients with urinary tract infection: implications on empiric therapy," Annals of Tropical Medicine and Public Health, vol. 5, no. 3, p. 181, 2012.

[6] B. Foxman, "Urinary tract infection syndromes," Infectious Disease Clinics of North America, vol. 28, no. 1, pp. 1-13, 2014.

[7] B. Foxman, "The epidemiology of urinary tract infection," Nature Reviews Urology, vol. 7, no. 12, pp. 653-660, 2010.

[8] P. Khandelwal, S. N. Abraham, and G. Apodaca, "Cell biology and physiology of the uroepithelium," American Journal of Physiology - Renal Physiology, vol. 297, no. 6, pp. F1477F1501, 2009.

[9] M. Dommergues, F. Daikha-Dahmane, F. Muller, M. C. Aubry, S. Lortat-Jacob, and C. Nihoul-Fékété, "Kidney and urinary tract disorders," Fetal Medicine E-Book: Basic Science and Clinical Practice, vol. 459, 2008.

[10] T. M. Hooton, S. F. Bradley, D. D. Cardenas et al., "Diagnosis, prevention, and treatment of catheter-associated urinary tract infection in adults: 2009 international clinical practice guidelines from the infectious diseases society of America," Clinical Infectious Diseases, vol. 50, no. 5, pp. 625-663, 2010.

[11] K. Gupta, T. M. Hooton, and W. E. Stamm, "Increasing antimicrobial resistance and the management of uncomplicated community-acquired urinary tract infections," Annals of Internal Medicine, vol. 135, no. 1, pp. 41-50, 2001.

[12] F. Ashraf, S. S. Iram, F. Rasheed, and M. Shaukat, "Comparison between non-catheterized and catheter-associated urinary tract infections caused by extended spectrum B-lactamase producing Escherichia coli and Klebsiella pneumoniae," bioRxiv, vol. 4, Article ID 019026, 2015.

[13] J. P. Horcajada, E. Shaw, B. Padilla et al., "Healthcare-associated, community-acquired and hospital-acquired bacteraemic urinary tract infections in hospitalized patients: a prospective multicentre cohort study in the era of antimicrobial resistance," Clinical Microbiology and Infections, vol. 19, no. 10, pp. 962-968, 2013.

[14] M. Fagan, M. Lindbæk, N. Grude et al., "Antibiotic resistance patterns of bacteria causing urinary tract infections in the elderly living in nursing homes versus the elderly living at home: an observational study," BMC Geriatrics, vol. 15, no. 1, p. 98, 2015.

[15] U. Kamat, A. Fereirra, D. Amonkar, D. Motghare, and M. Kulkarni, "Epidemiology of hospital acquired urinary tract infections in a medical college hospital in Goa," Indian Journal of Urology, vol. 25, no. 1, p. 76, 2009.

[16] C. King, L. Garcia Alvarez, A. Holmes, L. Moore, T. Galletly, and P. Aylin, "Risk factors for healthcare-associated urinary tract infection and their applications in surveillance using hospital administrative data: a systematic review," Journal of Hospital Infection, vol. 82, no. 4, pp. 219-226, 2012.

[17] G. Yismaw, D. Asrat, Y. Woldeamanuel, and C. G. Unakal, "Urinary Tract Infection: bacterial etiologies, drug resistance profile and associated risk factors in diabetic patients attending Gondar University Hospital, Gondar, Ethiopia," European Journal of Experimental Biology, vol. 2, no. 4, pp. 889-898, 2012.

[18] A. A. Abejew, A. A. Denboba, and A. G. Mekonnen, "Prevalence and antibiotic resistance pattern of urinary tract bacterial infections in Dessie area, North-East Ethiopia," BMC Research Notes, vol. 7, no. 1, p. 687, 2014.

[19] A. Derbie, D. Hailu, D. Mekonnen, B. Abera, and G. Yitayew, "Antibiogram profile of uropathogens isolated at Bahir dar regional health research laboratory center, northwest Ethiopia," The Pan African Medical Journal, vol. 26, 2017.
[20] N. Graves, E. Tong, A. P. Morton et al., "Factors associated with health care-acquired urinary tract infection," American Journal of Infection Control, vol. 35, no. 6, pp. 387-392, 2007.

[21] L. M. Irenge, L. Kabego, O. Vandenberg, R. B. Chirimwami, and J.-L. Gala, "Antimicrobial resistance in urinary isolates from inpatients and outpatients at a tertiary care hospital in South-Kivu Province (Democratic Republic of Congo)," BMC Research Notes, vol. 7, no. 1, p. 374, 2014.

[22] E.-M. M. Abubakar, "Antimicrobial susceptibility pattern of pathogenic bacteria causing urinary tract infections at the Specialist Hospital, Yola, Adamawa State, Nigeria," Journal of clinical medicine and research, vol. 1, no. 1, pp. 1-8, 2009.

[23] S. Manikandan, S. Ganesapandian, M. Singh, and A. Kumaraguru, "Antimicrobial susceptibility pattern of urinary tract infection causing human pathogenic bacteria," Asian Journal of Medical Sciences, vol. 3, no. 2, pp. 56-60, 2011.

[24] M. Bonadio, M. Meini, P. Spitaleri, and C. Gigli, "Current microbiological and clinical aspects of urinary tract infections," European Urology, vol. 40, no. 4, pp. 439-445, 2001.

[25] Clinical and Laboratory Standards Institute, Performance Standards for Antimicrobial Susceptibility Testing $29^{\text {th }}$, Clinical and Laboratory Standards Institute, Wayne, PA, USA, 2019.

[26] M. Cheesbrough, District Laboratory Practice in Tropical Countries, Cambridge University Press, Cambridge, UK, 2006.

[27] M. Amin, M. Mehdinejad, and Z. Pourdangchi, "Study of bacteria isolated from urinary tract infections and determination of their susceptibility to antibiotics," Jundishapur Journal of Microbiology, vol. 2, no. 3, p. 118, 2009.

[28] W. D. Seifu and A. D. Gebissa, "Prevalence and antibiotic susceptibility of Uropathogens from cases of urinary tract infections (UTI) in Shashemene referral hospital, Ethiopia," BMC Infectious Diseases, vol. 18, no. 1, p. 30, 2018.

[29] T. Gezmu, B. Regassa, A. Manilal, M. Mama, and B. Merdekios, "Prevalence, diversity, and antimicrobial resistance of bacteria isolated from the UTI patients of Arba Minch Province, southern Ethiopia," Translational Biomedicine, vol. 7, p. 3, 2016.

[30] L. Nesher, V. Novack, K. Riesenberg, and F. Schlaeffer, "Regional community-acquired urinary tract infections in Israel: diagnosis, pathogens, and antibiotic guidelines adherence: a prospective study," International Journal of Infectious Diseases, vol. 11, no. 3, pp. 245-250, 2007.

[31] A. Singhal, R. Sharma, M. Jain, and L. Vyas, "Hospital and community isolates of uropathogens and their antibiotic sensitivity pattern from a tertiary care hospital in north west India," Annals of Medical and Health Sciences Research, vol. 4, no. 1, pp. 51-56, 2014.

[32] S. Ahmad, "Pattern of urinary tract infection in Kashmir and antimicrobial susceptibility," Bangladesh Medical Research Council Bulletin, vol. 38, no. 3, pp. 79-83, 2012.

[33] U. Parvin and B. Mymensingh, Comparative Study Among the Bacterial Causes of Community-Acquired and Nosocomial Urinary Tract Infection, p. 11, Department of Microbiology Mymensingh Medical College, Mymensingh,. Bangladesh, 2009.

[34] M. Alo, A. Saidu, U. Ugah, and M. Alhassan, "Prevalence and antbiogram of bacterial isolates causing urinary tract infections at federal teaching hospital abakaliki I (FETHA I)," British Microbiology Research Journal, vol. 8, no. 2, pp. 403417, 2015.

[35] S. Nwadioha, E. Nwokedi, G. Jombo, E. Kashibu, and O. Alao, "Antibiotics susceptibility pattern of uropathogenic bacterial 
isolates from community and hospital-acquired urinary tract infections in a Nigerian Tertiary Hospital," The Internet Journal of Infectious Diseases, vol. 8, no. 1, pp. 001-008, 2010.

[36] I. Abdulahi and S. Gebre-Selassie, Common Bacterial Pathogens and Their Antimicrobial Susceptibility Patterns in $\mathrm{Pa}$ tients with Symptomatic Urinary Tract Infections, Hiwot-Fana and Jugal Hospitals, Harar City, Eastern Ethiopia, 2011.

[37] S. Melaku, M. Kibret, B. Abera, and S. Gebre-Sellassie, "Antibiogram of nosocomial urinary tract infections in Felege Hiwot referral hospital, Ethiopia," African Health Sciences, vol. 12, no. 2, pp. 134-139, 2012.

[38] M. Gizachew, M. Kebede, Y. Merid, Y. Sinshaw, M. Tiruneh, and M. Alemayehu, "Escherichia coli isolated from patients suspected for urinary tract infections in Hawassa Referral Hospital, Southern Ethiopia: an institution-based cross-sectional study," Journal of Microbiology Research, vol. 1, pp. 9-15, 2013.

[39] O. O. Timothy, F. J. Olusesan, B. O. Adesola, A. A. Temitayo, F. O. David, and O. O. Ige, "Antibiotic resistance pattern of bacterial isolates from cases of urinary tract infections among hospitalized and outpatients at a tertiary health facility in South Western Nigeria," Annals of Tropical Medicine and Public Health, vol. 7, no. 2, p. 130, 2014.

[40] M. Kibret and B. Abera, "Prevalence and antibiogram of bacterial isolates from urinary tract infections at Dessie Health Research Laboratory, Ethiopia," Asian Pacific journal of tropical biomedicine, vol. 4, no. 2, pp. 164-168, 2014.

[41] K. Iregbu and P. Nwajiobi-Princewill, "Urinary tract infections in a tertiary hospital in Abuja, Nigeria," African Journal of Clinical and Experimental Microbiology, vol. 14, no. 3, pp. 169-173, 2013.

[42] T. AlZubiery, T. Alharazi, H. Alsumairy, A. Al-Zubeiry, A. Yusr, and A. L. Al-shami Huda, "Current antibiotic sensitivity pattern of clinically isolated Klebsiella pneumonia," Saudi Journal of Biomedical Research, vol. 3, no. 2, pp. 23-32, 2018.

[43] L. Nimri, Community-acquired Urinary Tract Infections in a Rural Area in Jordan: Predominant Uropathogens, and Their Antimicrobial Resistance. 2010.

[44] S. Eshetie, C. Unakal, A. Gelaw, B. Ayelign, M. Endris, and F. Moges, "Multidrug resistant and carbapenemase producing Enterobacteriaceae among patients with urinary tract infection at referral Hospital, Northwest Ethiopia," Antimicrobial Resistance and Infection Control, vol. 4, no. 1, p. 12, 2015.

[45] S. B. Gadadavar and N. N. Patil, "Association of host characters and uropathogens in community and hospital-acquired urinary tract infection," Indian Journal of Preventive and Social Medicine, vol. 4, pp. 352-357, 2015.

[46] F. Moges, G. Mengistu, and A. Genetu, "Multiple drug resistance in Urinary pathogens at Gondar College of Medical science hospital, Ethiopia," East African Medical Journal, vol. 79, no. 8, pp. 415-420, 2002.

[47] S. Becattini, Y. Taur, and E. G. Pamer, "Antibiotic-induced changes in the intestinal microbiota and disease," Trends in Molecular Medicine, vol. 22, no. 6, pp. 458-478, 2016.

[48] M. Odoki, A. Almustapha Aliero, J. Tibyangye et al., "Prevalence of bacterial urinary tract infections and associated factors among patients attending hospitals in bushenyi district, Uganda," International journal of microbiology, vol. 2019, Article ID 4246780, 8 pages, 2019.

[49] B. Oluremi, A. Idowu, and J. Olaniyi, "Antibiotic susceptibility of common bacterial pathogens in urinary tract infections in a Teaching hospital in South-western Nigeria,"
African Journal of Microbiology Research, vol. 5, no. 22, pp. 3658-3663, 2011.

[50] A. Smithson, J. Ramos, M. T. Bastida et al., "Differential characteristics of healthcare-associated compared to community-acquired febrile urinary tract infections in males," European Journal of Clinical Microbiology \& Infectious Diseases, vol. 34, no. 12, pp. 2395-2402, 2015.

[51] E. Hopps, A. Camera, and G. Caimi, "Polimorfonucleatie malattia diabetica," Minerva Medica, vol. 99, no. 2, pp. 197202, 2008

[52] K. Yadav and S. Prakash, "Antimicrobial resistance pattern of uropathogens causing urinary tract infection (UTI) among diabetics," Biomedical Research International, vol. 1, pp. 7-15, 2016.

[53] This manuscript presents in the <research square $>$ as a preprint.https://www.researchsquare.com/article/rs-157817/ v1. 